The hexagon of carbon that makes up a nanotube has a predilection for clinging to other hexagons. One of the many challenges of working with CNTs is that they tend to stick to each other. Attaching a molecule to the sidewall of a nanotube serves a double purpose: It stops nanotubes from sticking, so that they can be processed and manipulated more easily, and it allows researchers to control and change the electronic properties of the nanotubes. Still, most such molecules also destroy the CNTs' conductance because they make the nanotube structurally more similar to diamond, which is an insulator, rather than to graphite, a semimetal.

The researchers indicate that their studies show that carbenes and nitrenes work by breaking a molecular bond on the nanotube's wall while creating their own new bond to the nanotube. This process-one bond formed, one bond lost-restores the perfect number of bonds each carbon atom had in the original nanotube; thus, "conductance is recovered," Marzari said.

The theory indicates that some molecular handles can even transform between a "bond-broken" and a "bond-intact" state, allowing the CNTs to act like switches that can be turned on or off in the presence of certain substances or with a laser beam.

"This direct control of conductance may lead to novel strategies for the manipulation and assembly of nanotubes in metallic interconnects, or to sensing or imaging devices that respond in real time to optical or chemical stimuli," Marzari said.

The next step is for experiments to confirm that the approach works.

\section{Model of Collagen Nanostructure Explains Its Strength}

Collagen's characteristic nanostructure may be the reason for its high strength and ability to sustain large deformation in its physiological role in tissues such as bone, tendon, and muscle. Previous experimentation has shown that collagen isolated from different tissue sources universally displays a design that consists of a staggered assembly of tropocollagen molecules with lengths of approximately $300 \mathrm{~nm}$. The reason why strands of amino acids associate to form tropocollagen molecules consistently at this length has been an unexplained phenomenon.

M.J. Buehler, principal investigator at the Atomistic Mechanics Modeling Laboratory at the Massachusetts Institute of Technology, has used a combination of theoretical and molecular modeling that led to a breakthrough in understanding how molecular and tissue properties are linked. Buehler has reported his findings in the August 15 issue of the Proceedings of the National Academy of Sciences (p. 12285; DOI: 10.1073/pnas.0603216103).

Buehler discovered that the characteristic design of collagen displays a clever strategy that enables nature to take advantage of the nanoscale properties of individual molecules at larger scales, leading to a tough material. This is achieved by arranging tropocollagen molecules into a staggered assembly known as collagen fibrils, tiny fibers with diameters of 50-200 nm and lengths of several micrometers. When a tensile force is applied at the end of a collagen fibril, the force is transmitted as shear forces between molecules and a tensile force within molecules. Whereas the elastic tensile strength of the tropocollagen mol-

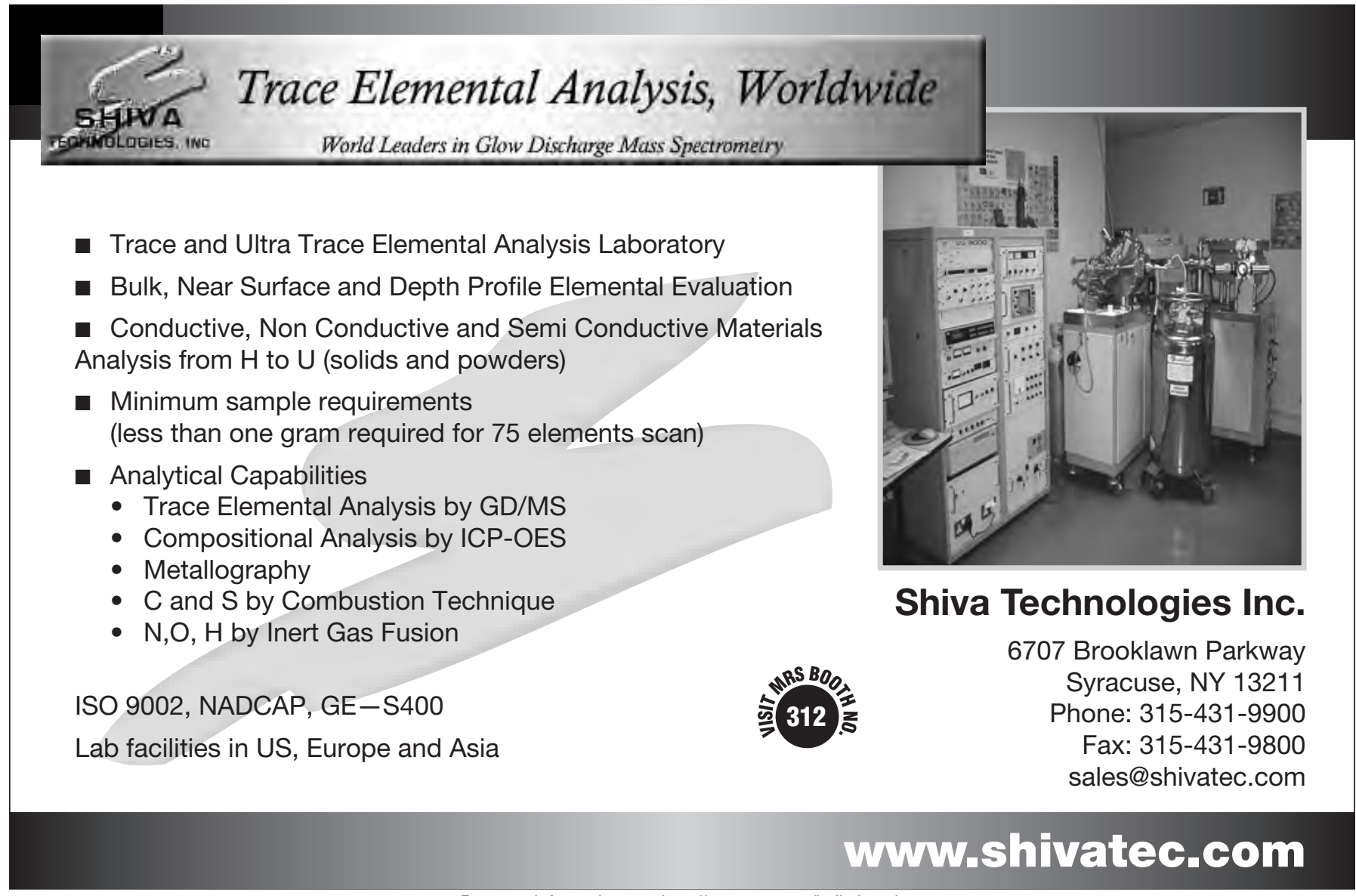

For more information, see http://www.mrs.org/bulletin_ads 
ecules stems from their triple-helical arrangements, the shear force between molecules is transmitted by a combination of weak interactions, including hydrogen bonds and dispersive interactions, as well as some covalent cross-links (see Figure 1).

Individual tropocollagen molecules are 5-10 times stronger than steel and able to
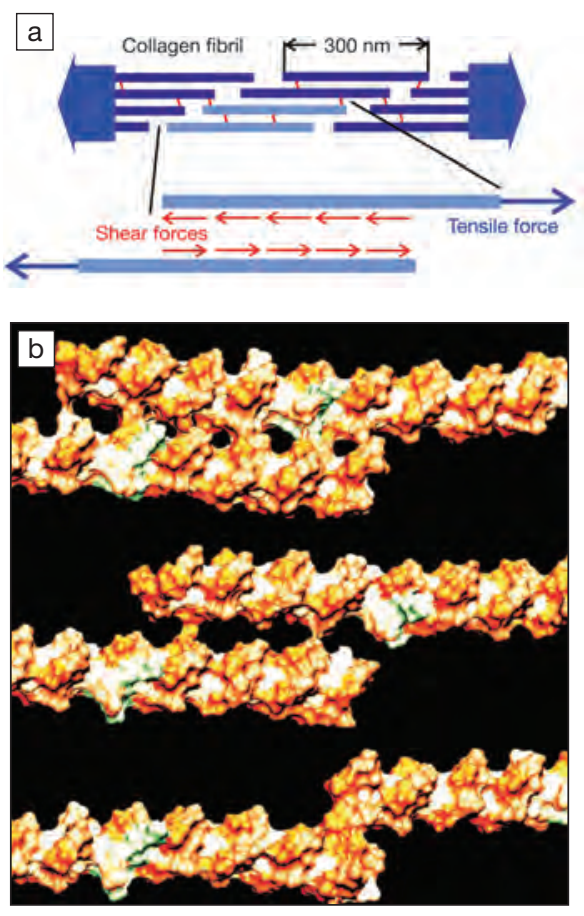

Figure 1. (a) (top) Staggered arrangement of tropocollagen molecules into collagen fibrils. (bottom) Expanded view illustrates how forces are transmitted inside the collagen fibril as a result of macroscopic external tensile loading. When a tensile force is applied at the end of a collagen fibril, the force is transmitted as a shear force between molecules and a tensile force within molecules. (b) Sequence of images displaying the atomistic mechanisms as two tropocollagen molecules in a collagen fibril undergo nanoscopic shear deformation. This intermolecular shear deformation is induced by macroscopic tensile loading. The sequence depicts the surface of each tropocollagen molecule and its interaction with other molecules. Tropocollagen molecules interact through a nanoscale layer of water molecules and weak interactions, including hydrogen bonds and electrostatic forces. These atomic interactions break as molecules slide on top of each other. (Figure courtesy of M.J. Buehler,http://web.mit.edu/mbuehler/ www/, rendered using visual molecular dynamics [VMD] software.) sustain tensile strains of up to $50 \%$ before fracture occurs. Steel, in comparison, typically sustains tensile strains of only a few percent before it breaks. Buehler said, "The fracture strength of individual [tropocollagen] molecules is largely controlled by covalent polypeptide chemistry. The shear strength between two [tropocollagen] molecules is controlled by weak dispersive and hydrogen-bond interactions and by some intermolecular covalent cross-links."
"The natural design of collagen represents a delicate balance between tensile forces within each tropocollagen molecule and shear forces between the molecules," said Buehler. He discovered that there exists a characteristic molecular length scale at which collagen fibrils have optimal mechanical properties-being strong while providing large energy dissipation. Buehler's modeling results predict that the optimal length of tropocollagen molecules is approximately $200 \mathrm{~nm}$,

\section{Cost-Effective Portable Spin Coater}

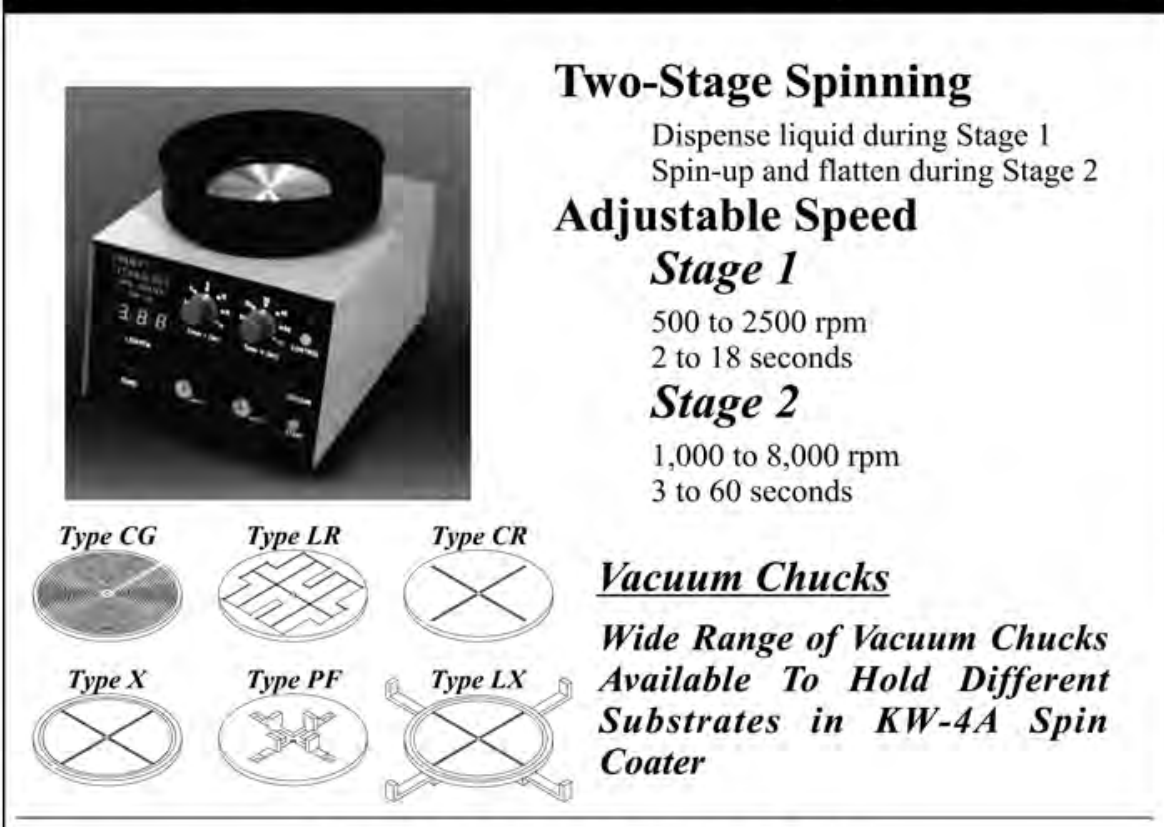

\section{KW-4A SERIES PRODUCT LINE}
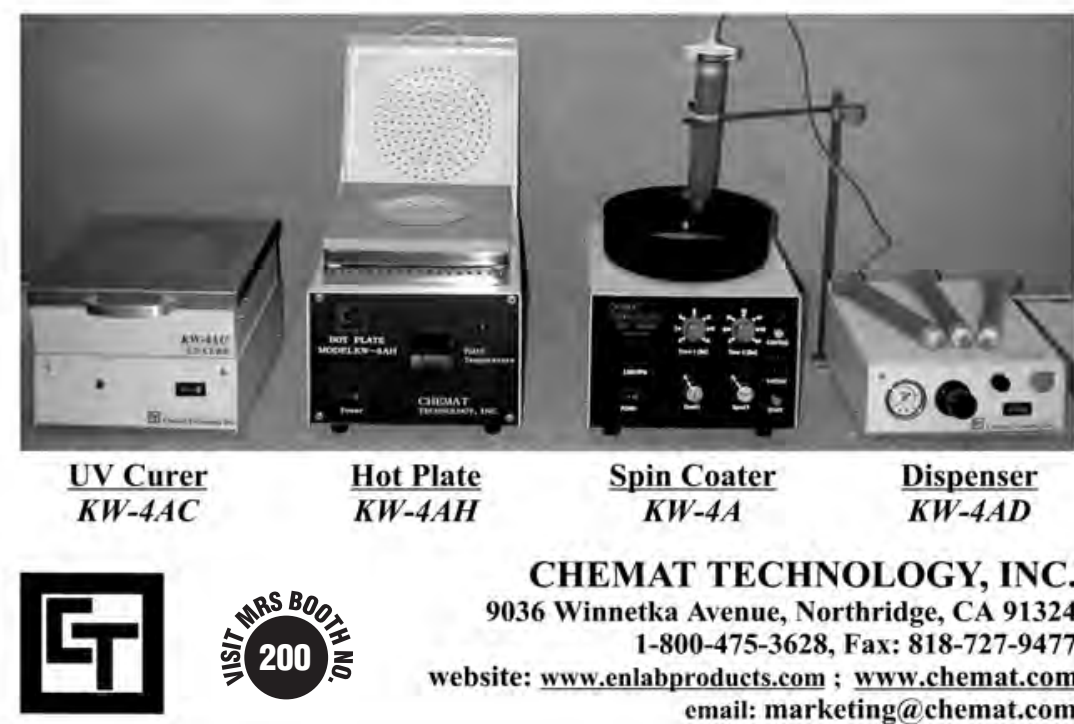

CHEMAT TECHNOLOGY, INC.

9036 Winnetka Avenue, Northridge, CA 91324

1-800-475-3628, Fax: 818-727-9477

200 website: www.enlabproducts.com ; www.chemat.com email: marketing@chemat.com

For more information, see http://www.mrs.org/bulletin_ads 
which is very close to the experimentally observed characteristic length of $280 \mathrm{~nm}$. Buehler developed a new theoretical framework that provides a quantitative treatment of the nanomechanics of collagen fibrils.

Buehler used large-scale computer simulations that begin at the atomistic scale, treating individual chemical interactions based on quantum mechanical calculations to obtain results consistent with his theory. Previous models of collagen typically involved empirical parameters and lacked a rigorous connection between quantum chemistry, molecular structure, material properties and collagen's physiological function. Buehler's model provides a first-principles-based materials representation. Furthermore, the model provides a basis for understanding the effects of diseases "caused by defects in the molecular structure of collagen altering the intermolecular and molecular properties due to genetic mutations, which modifies the mechanical behavior of collagen fibrils."

Collagen, an extracellular matrix protein, plays an important role in defining the infrastructure of physiological tissues under load or strain, and is critical to tissues within the skeletal, muscular, and cardiovascular networks. Improved understanding of nature's design criteria will help guide materials and biomedical engineers to develop enhanced biomimetic polymers. Buehler's work could contribute to research that may one day develop cures for collagen-related diseases such as the Ehler-Danlos syndrome, joint hyperextensibility, or scurvy.

"We are currently extending our models to describe the deformation mechanics of other structural proteins and more complex materials such as bone," said Buehler. "For example, the smallest building blocks of bone are mineralized collagen fibrils, which can be modeled using similar concepts."

\section{Combination of Top-Down and Bottom-Up Approaches Allows Fabrication of High-Aspect-Ratio 2D KTiOPO ${ }_{4}$ Photonic Crystals}

In many applications where optoelectronic modulation of light is needed, it would be very useful to have micro- or nanostructures fabricated from materials with large electro-optic coefficients such as the ones found in many inorganic nonlinear crystals. Unfortunately, when topdown procedures are applied to such materials to produce, for instance, twodimensional (2D) photonic crystals, they are not as effective as when they are applied to semiconductors. A. Peña of the Universitat Rovira i Virgili, S. Di Finizio from the ICFO-Institut de Ciencies Fotoniques, J. Martorell and A. Rodriguez of the Universitat Politecnica de Catalunya, Spain, and their colleagues have obtained 2D photonic crystals by growing microrods of $\mathrm{KTiOPO}_{4}$ inside the air holes of an ordered macroporous silicon membrane closely bound to a $\mathrm{KTiOPO}_{4}$ substrate (see Figure 1).

As reported in the September issue of Advanced Materials (DOI: 10.1002/adma. 200502566), the researchers prepared the silicon membranes by computer-controlled light-assisted electrochemical etching of a patterned $n$-type $<100>$ silicon wafer, with which they control the growth, size, depth, and quality of the pores. A c-oriented $\mathrm{KTiOPO}_{4}$ substrate was bound to the sili-

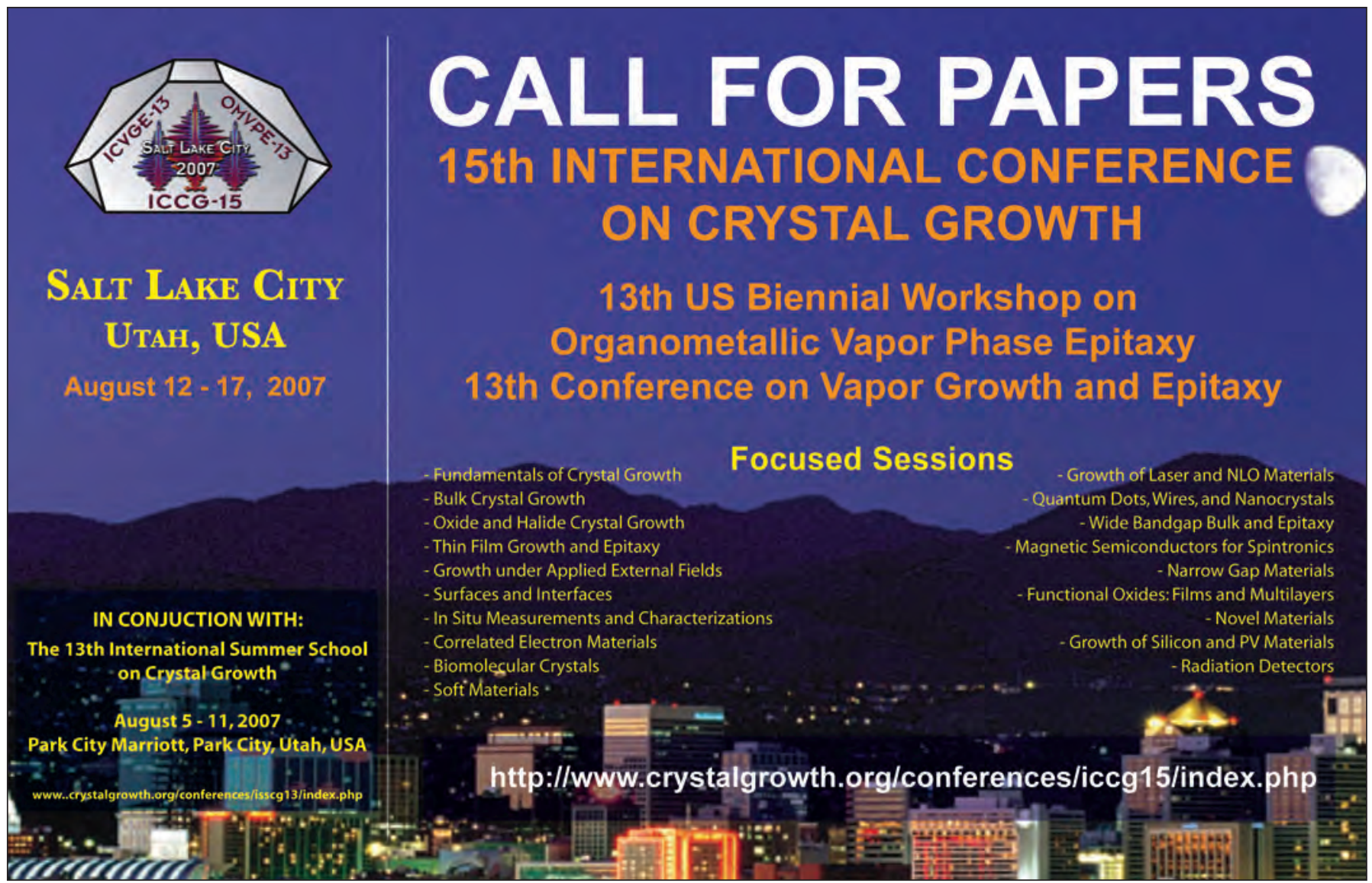

For more information, see http://www.mrs.org/bulletin_ads 